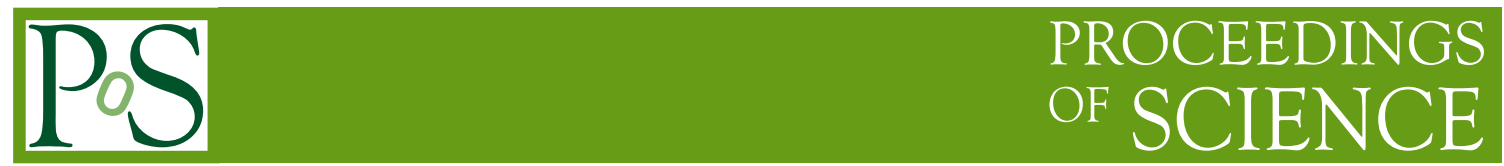

\title{
Hidden sector dark matter and Higgs physics
}

\author{
Seungwon Baek, Pyungwon Ko*, Wand-II Park and Eibun Senaha \\ School of Physics, KIAS, Seoul 130-722, Korea \\ E-mail: pko@kias.re.kr
}

\begin{abstract}
We consider a hidden sector dark matter, where a singlet fermion is a cold dark matter and a real singlet scalar boson $S$ is a messenger between the SM and the hidden sectors. This singlet scalar will mix with the SM Higgs boson $h$, and we expect there are two Higgs-like scalar bosons $H_{1}$ and $H_{2}$. Imposing all the relevant constraints from collider search bounds on Higgs boson, DM scattering cross section on proton and thermal relic density. We find that there is a destructive interference between $H_{1}$ and $H_{2}$ contributions to the direct detection cross section of the DM. Also one of the two Higgs-like scalar bosons can easily escape the detections at the LHC, and there will be a universal reduction of the signal strength for the observed $125 \mathrm{GeV}$ Higgs-like boson, which could be tested at the LHC with more data in the future.
\end{abstract}

VIII International Workshop on the Dark Side of the Universe,

June 10-15, 2012

Rio de Janeiro, Brazil

*Speaker. 


\section{Motivations}

The standard model (SM) has been tested by many experiments from atomic scale up to electroweak scale, and was extremely successful. However, there are three observational facts which call for new physics beyond the SM:

- Baryon number asymmetry of the universe (BAU)

- Neutrino masses and mixings

- Nonbaryonic Cold dark matter (CDM).

There are many models and suggestions for each problem listed above. The most economical way to solve the BAU and the neutrino masses and mixings is probably to introduce heavy righthanded neutrinos and invoke seesaw mechanism. Leptogenesis can turn to baryogenesis around electroweak phase transition by sphalerons.

Given the triumphant success of the SM with its aforementioned shortcomings kept in mind, the most important questions in particle physics at the LHC era would be the following:

- Origin of electroweak symmetry breaking (EWSB)

- Nature of cold dark matter (CDM)

- Origin of flavor structure and families.

Any new physics models at the electroweak scale are strongly constrained by electroweak precision test and CKM phenomenology, if new physics feels the SM gauge interactions. In this case, the new physics scales should be larger than $O(1) \mathrm{TeV}$ and $O(100) \mathrm{TeV}$ in order to be safe from the EWPT and CKM phenomenology, respectively. On the other hand, fine tuning problem of (Higgs mass) $)^{2}$ requires that new physics scale should be around $\lesssim O(1) \mathrm{TeV}$. Thus there is strong tension between two.

On the other hand, if new physics particles are neutral under the SM gauge group and do not feel SM gauge interactions, the constraints from the EWPT and CKM fit can be relaxed by a significant amount, and new physics scales could be easily at the EW scale. Thus we are led to consider a weak scale hidden sector which is neutral under the SM gauge interaction. The hidden sector matters can be natural CDM if there are suitable messengers between the SM and the hidden sectors. In this talk, I will discuss singlet fermion hdden sector dark matters based on the works $[1,2]$. (Another interesting case where the hidden sector gauge interaction is strong and confining like the ordinary QCD is discussed in Ref.s $[3,4,5]$.) In this approach, there is no resolution of fine tuning problem of (Higgs mass) ${ }^{2}$ parameter, since new particles do not carry the SM gauge quantum numbers. And I don't address the fine tuning problem in this talk.

\section{Hidden sector singlet fermion DM model}

\subsection{Model}

Let us consider a singlet fermion dark matter $\psi$ with a real singlet scalar messenger $S$ with the following lagrnagian [1]:

$$
\mathscr{L}=\mathscr{L}_{\mathrm{SM}}+\mathscr{L}_{\text {hidden }}+\mathscr{L}_{\text {portal }}
$$


where

$$
\begin{aligned}
\mathscr{L}_{\text {hidden }} & =\frac{1}{2}\left(\partial_{\mu} S \partial^{\mu} S-m_{S}^{2} S^{2}\right)-\mu_{S}^{3} S-\frac{\mu_{S}^{\prime}}{3} S^{3}-\frac{\lambda S}{4} S^{4}+\bar{\psi}\left(i \not \partial-m_{\psi_{0}}\right) \psi-\lambda S \bar{\psi} \psi, \\
\mathscr{L}_{\text {portal }} & =-\mu_{H S} S H^{\dagger} H-\frac{\lambda_{H S}}{2} S^{2} H^{\dagger} H,
\end{aligned}
$$

We assume $\psi$ carries a conserved dark charge, and is distinguished from the right-handed neutrinos.

In the literature, the Higgs portal fermion dark matter is often discussed using the following effective lagrangian :

$$
\mathscr{L}_{\text {portal }}=-\bar{\psi}\left(m+\lambda_{\psi H} \frac{H^{\dagger} H}{\Lambda}\right) \psi
$$

There are two scalar bosons in our model ( $h$ and $s$ ), and we will find that the physics results from our model are very different from those based on Eq. (2.4).

\subsection{Constraints}

We consider the following constraints on the model parameters:

- the perturbative unitarity condition on the Higgs sector

- the LEP bound on the SM Higgs boson mass

- the oblique parameters $S, T$ and $U$ obtained from the EWPT

- the observed CDM density, $\Omega_{\mathrm{CDM}} h^{2}=0.1123 \pm 0.0035$ which we assume is saturated by the thermal relic $\psi$,

- the upper bound on the DM-proton scattering cross section obtained by the XENON100 experiment.

Note that the first three constraints are independent of the dark matter sector, and they apply to the SM plus a singlet scalar model without dark matter as well.

The extended Higgs sector gives extra contribution to the gauge boson self-energy diagrams, as the SM Higgs boson does. This can affect the EWPT leading to the constraints on the oblique parameters, $S, T$ and $U$, by the Higgs sector. It turns out that the EWPT constraint on our model is generically much less severe than on the SM. The SM always predicts a negative $\Delta T$ for the Higgs mass larger than $m_{h}=120 \mathrm{GeV}$. However, $\Delta T$ can be either positive or negative in our model, and the mixing between the singlet scalar is fine with the EWPT (see Fig. 1).

\subsection{Dark matter phenomenology}

The observed DM relic density, $\Omega_{\mathrm{CDM}} h^{2} \simeq 0.1123 \pm 0.0035$ is related with the thermally averaged annihilation cross section times relative velocity at freeze-out temperature. The annihilation cross section of a DM pair is proportional to $\sin ^{2} 2 \alpha$. Since the EWPT and LHC observation of the SM-like Higgs boson restricts $\alpha$ to be small, the cross section is generically much smaller than is needed to explained the current relic density. This can be seen in Fig. 2 except for resonance regions. 


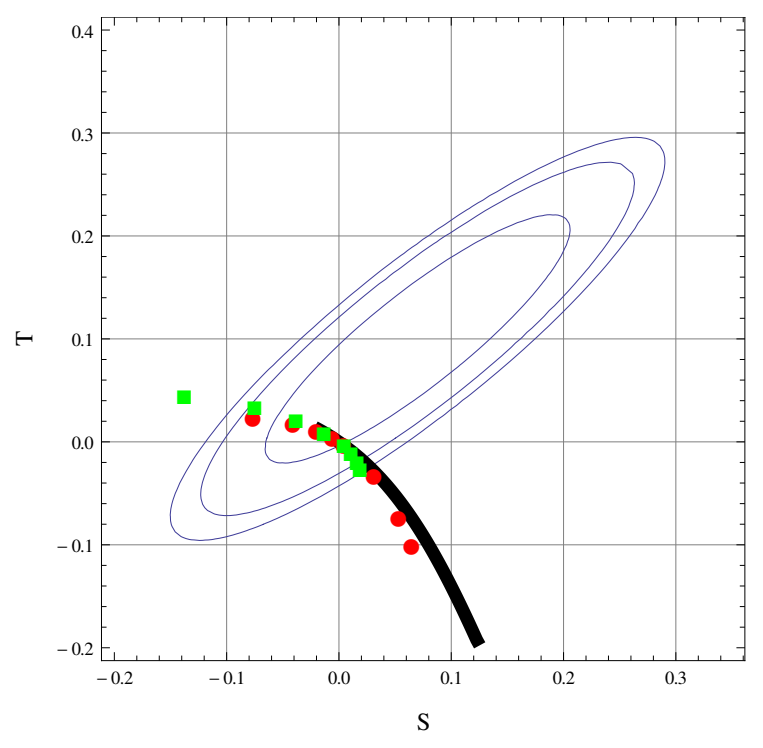

Figure 1: The prediction of $(S, T)$ parameters. We fixed the reference Higgs mass to be 120 $\mathrm{GeV}$. The ellipses are $(68,90,95) \% \mathrm{CL}$ contours from the global fit. The thick black curve shows the SM prediction with the Higgs boson mass in the region $(100,720) \mathrm{GeV}$. The red, green dots correspond to $\alpha=45^{\circ}, 20^{\circ}$, respectively. The dots are for the choices $\left(m_{1}, m_{2}\right)(\mathrm{GeV})=$ $(25,125),(50,125),(75,125),(100,125),(125,125),(125,250),(125,500),(125,750)$ from above for each color.

We used the micrOMEGAs package for numerical calculation of DM relic density and direct detection cross section. In Fig. 2, we show the CDM relic density as a function of $m_{2}$ for various choices of $m_{\psi}=100,500,1000,1500 \mathrm{GeV}$, with $\lambda=0.4$ and $\alpha=0.1$. We can always find out the $m_{2}$ value which can accommodate thermal relic density of the singlet fermion CDM $\psi$. Note that there is no strong constraint on the heavier Higgs with a small mixing angle $\alpha$, because $\mathrm{H}_{2}$ would be mostly a singlet scalar so that it is very difficult to produce it at colliders, and also it could decay into a pair of CDM's with a substantial branching ratio.

The dark matter scattering on proton target is given by

$$
\sigma_{p} \simeq 8.6 \times 10^{-9} \mathrm{pb}\left(\frac{125 \mathrm{GeV}}{m_{1}}\right)^{4}\left(1-\frac{m_{1}^{2}}{m_{2}^{2}}\right)^{2}\left(\frac{\lambda \sin \alpha \cos \alpha}{0.1}\right)^{2} .
$$

Note that there is a generic cancellation between the $H_{1}$ and $H_{2}$ contributions. Due to this cancellation, the constraint from direct detection of dark matter becomes much weaker on the Higgs couplings to the DM's. If we ignored the singlet scalar from the beginning (for example in the effective lagrangian approach using Eq. (2.4)), we could not enjoy such cancellation in $\sigma_{p}$, so that the Higgs coupling to the DM would be much more tightly constrained.

\subsection{Vacuum stability}

Compared with the SM, there are additional fields $\psi$ and $S$ that contribute to the effective potential for $H$ and $S$, and the vacuum stability can be modified from the SM case. We studied this issue very carefully in Ref. [2], and found that the SM vacuum can be stable up to Planck scale within this model (see Fig. 3). 


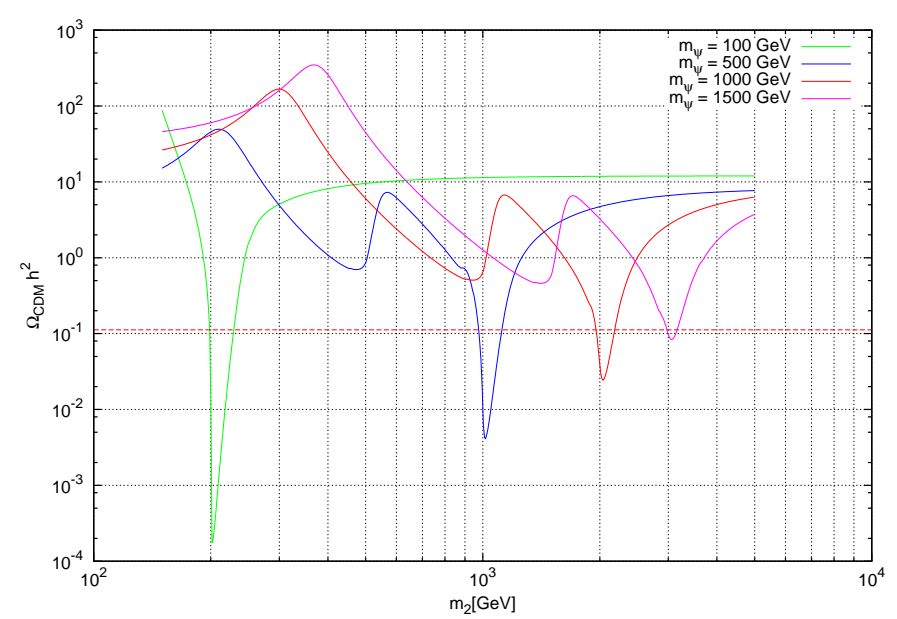

Figure 2: Dark matter thermal relic density $\left(\Omega_{\mathrm{CDM}} h^{2}\right)$ as a function of $m_{2}$ for $m_{1}=125 \mathrm{GeV}, \lambda=0.4$, $\alpha=0.1$ and $m_{\psi}=100,500,1000,1500 \mathrm{GeV}$ from top to bottom at right side. The dotted red line corresponds to the observed value, $\Omega_{\mathrm{CDM}} h^{2}=0.112$.
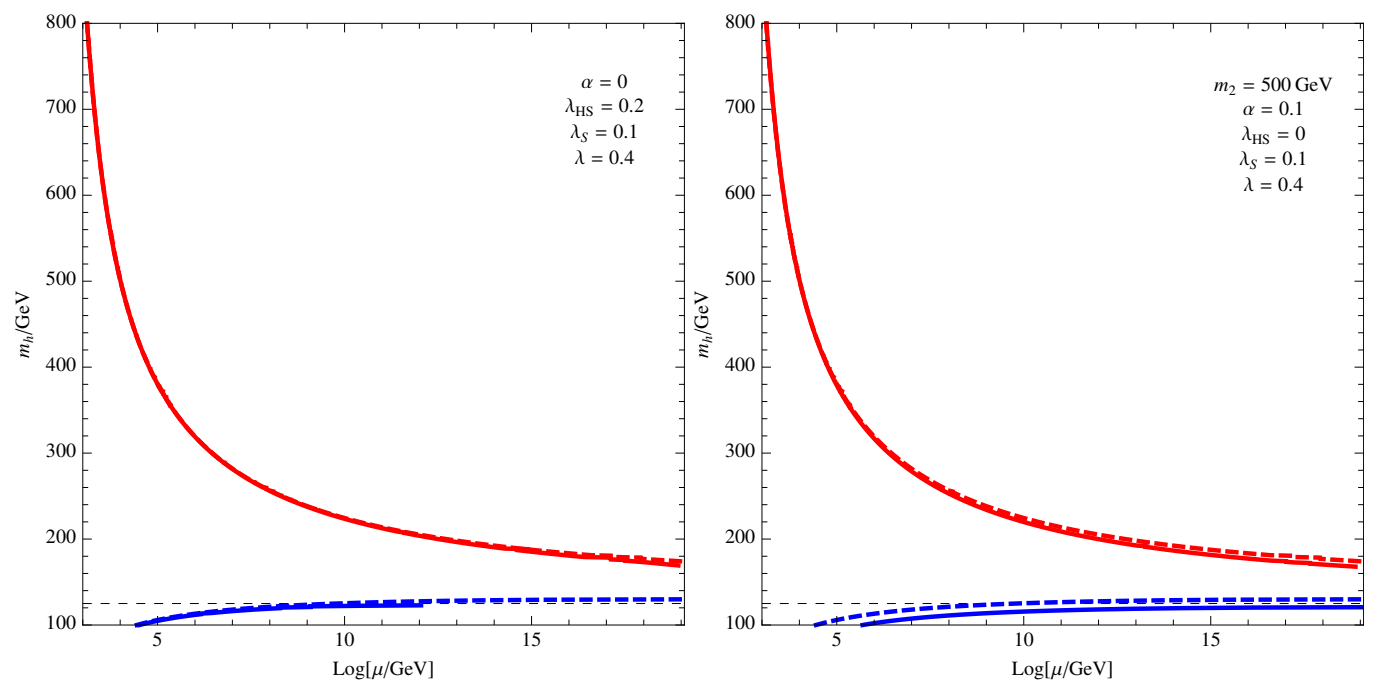

Figure 3: The mass bound of SM-like Higgs $\left(m_{1}\right)$ as a function of energy scale for $\left(\alpha, \lambda_{H S}\right)=$ $(0,0.2)$ (left), $(0.1,0)$ (right) with $\lambda_{S}=0.1$ and $\lambda=0.4$. The red/blue line corresponds to triviality/vacuumstability bound in SM(dashed) and our model(solid). The dashed black line corresponds to $m_{1}=125 \mathrm{GeV}$.

\section{Implications for the Higgs search at the LHC}

In this section, we investigate if it is possible to discover the Higgs(es) at the LHC, taking into account of all the constraints discussed in the previous section. The signal strength or "the reduction factor" in the event number of a specific final state SM particles, $X_{\mathrm{SM}}$, in the Higgs boson decays is defined as

$$
r_{i} \equiv \frac{\sigma_{H_{i}} B_{H_{i} \rightarrow X_{\mathrm{SM}}}}{\sigma_{H_{i}}^{\mathrm{SM}} B_{H_{i} \rightarrow X_{\mathrm{SM}}}^{\mathrm{SM}}}(i=1,2),
$$

where $\sigma_{H_{i}}$ and $B_{H_{i} \rightarrow X_{\mathrm{SM}}}$ are the production cross section of $H_{i}$, and the branching ratio of $H_{i} \rightarrow X_{\mathrm{SM}}$ 
respectively, while $\sigma_{H_{i}}^{\mathrm{SM}}$ and $B_{H_{i} \rightarrow X_{\mathrm{SM}}}^{\mathrm{SM}}$ are the corresponding quantities of the SM Higgs with mass $m_{i}$. Note that the signal strength $r_{i}$ becomes less than "1" due to the mixing between $h$ and $s$, even if the invisible mode $\left(H_{i} \rightarrow \psi \bar{\psi}\right)$ or the Higgs-splitting mode $\left(H_{2} \rightarrow H_{1} H_{1}\right)$ is kinematically forbidden in the Higgs decay. In other words, a reduced signal of the Higgs boson at the LHC would be a generic signature of the mixing of the SM Higgs boson with extra singlet scalar boson(s).

We study the following three benchmark scenarios classified according to the Higgs mass relations:

- Scenario 1 (S1): $m_{1} \sim 125 \mathrm{GeV} \ll m_{2}$

- Scenario 2 (S2): $m_{1} \sim m_{2} \sim 125 \mathrm{GeV}$

- Scenario 3 (S3): $m_{1} \ll m_{2} \sim 125 \mathrm{GeV}$

We scanned the remaining parameters in the range

$$
0<\lambda<1, \quad 10 \mathrm{GeV}<M_{\psi}<100 \mathrm{GeV}, \quad 0<\alpha<\pi / 2 .
$$

All the points in the plots satisfy the constraints described earlier.

We can divide the $\sigma_{p}$ (in $\mathrm{pb}$ ) into two regions:

$$
\sigma_{p}^{>}: 10^{-9}<\sigma_{p}<10^{-8}, \quad \sigma_{p}^{<}: \sigma_{p}<10^{-9},
$$

where the former region can be probed in near future direct search experiments. The relic density is also divided into two regions:

$$
\left(\Omega_{\mathrm{CDM}} h^{2}\right)^{3 \sigma}: 0.1018<\Omega_{\mathrm{CDM}} h^{2}<0.1228, \quad\left(\Omega_{\mathrm{CDM}} h^{2}\right)^{<}: \Omega_{\mathrm{CDM}} h^{2}<0.1018 .
$$

where the former is the WMAP $3 \sigma$ allowed region.

The region that the LHC at $14 \mathrm{TeV}$ can probe at $3 \sigma$ level with $5(10) \mathrm{fb}^{-1}$ luminosity is represented by solid (dashed) line. The $\mathrm{S} 1$ scenario can be tested fully at the LHC with $10 \mathrm{fb}^{-1}$ by observing $H_{1}$. In the case of S2 the LHC may see both Higgs bosons with the standard search strategy. However, there are still some points which the LHC has difficulty to find two Higgs bosons. These are the points near the origin $\left(r_{1} \approx r_{2} \approx 0\right)$ where the invisible decays becomes dominant. In S3 the region with small $r_{2}(<0.24)$ can not be probed with the standard decay channels. However, once $H_{2} \rightarrow H_{1} H_{1}$ is open, this region can also be tested at the LHC.

\section{Conclusions}

In this talk, I discussed an example of hidden sector dark matter model with a singlet fermion as a CDM, and discussed their interplay with phenomenology of the SM Higgs boson. Our results are completely different from those based on the effective lagrangian (2.4). The same is true for the Higgs portal vector dark matter [6].

Generic signatures of hidden sector fermion dark matter can be summarized as follows:

- Thermal relic density of hidden sector DM can be easily compatible with the WMAP observation, and they can be detected in the direct detection experiments. 

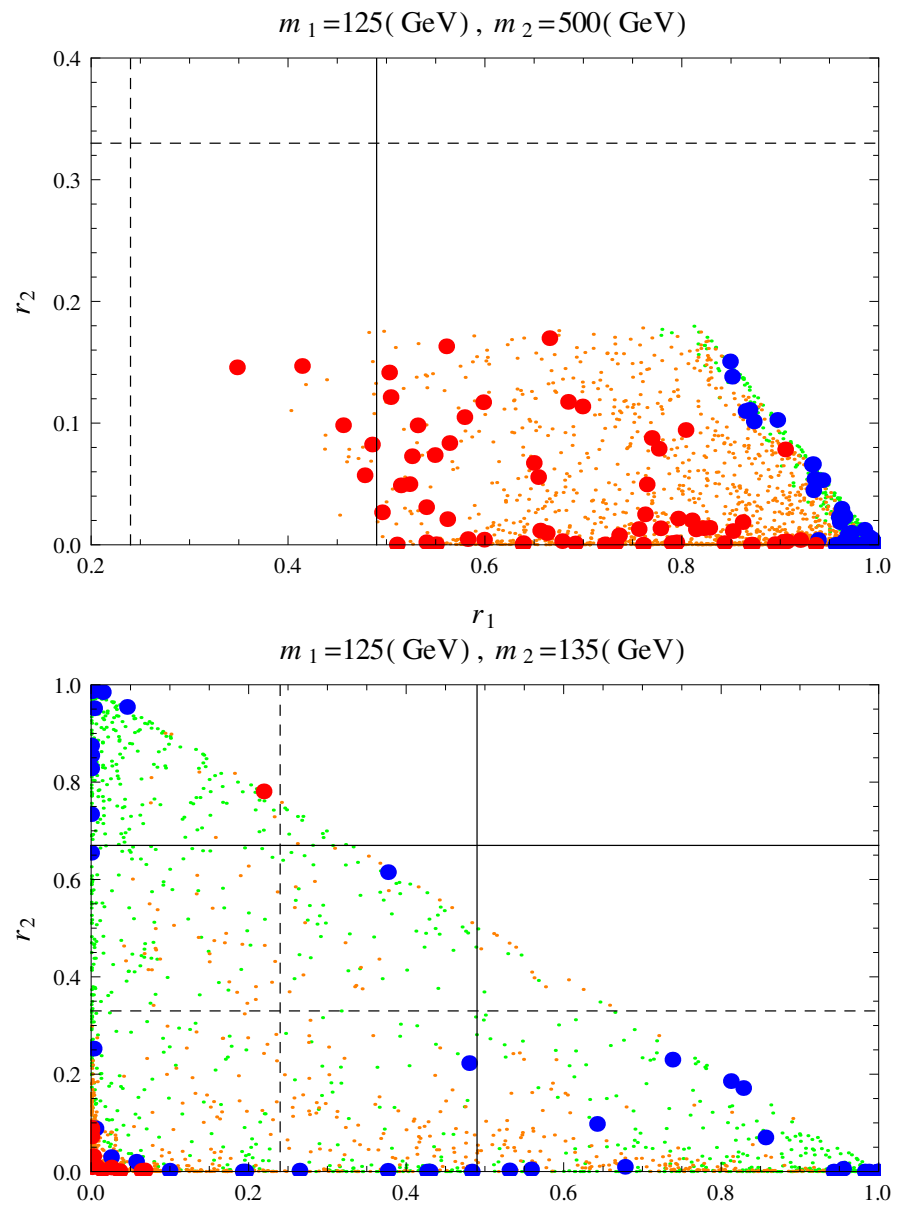

$r_{1}$

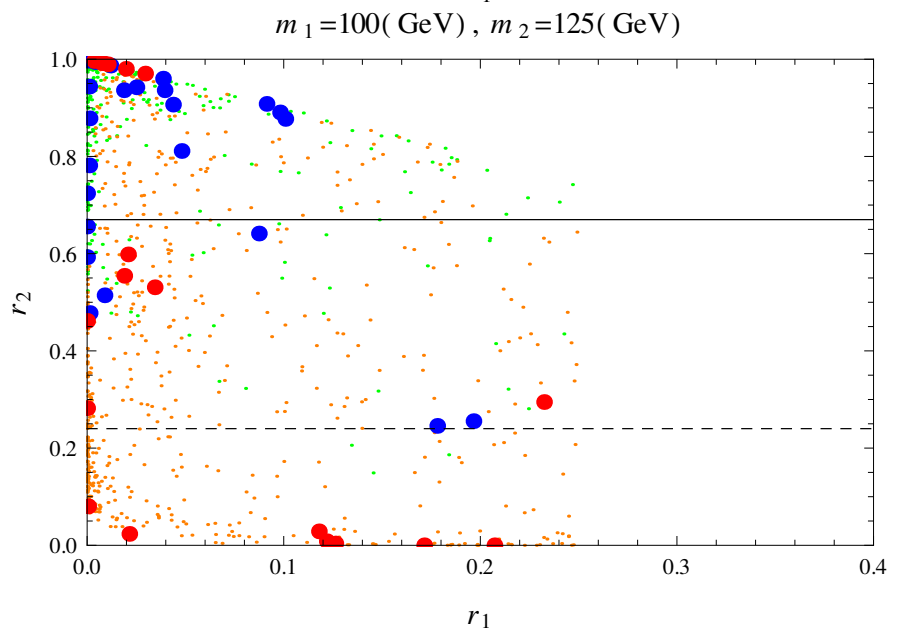

Figure 4: Scatter plot in $\left(r_{1}, r_{2}\right)$ plane for the scenario S1, S2 and S3 (from above). The region that the LHC can probe at $3 \sigma$ level with $5(10) \mathrm{fb}^{-1}$ luminosity is represented by solid (dashed) line. The points represent 4 different cases: $\left(\Omega_{\mathrm{CDM}} h^{2}\right)^{3 \sigma}, \sigma_{p}^{>}$(big red), $\left(\Omega_{\mathrm{CDM}} h^{2}\right)^{3 \sigma}, \sigma_{p}^{<}$(big blue), $\left(\Omega_{\mathrm{CDM}} h^{2}\right)^{<}, \sigma_{p}^{>}$(small orange), and $\left(\Omega_{\mathrm{CDM}} h^{2}\right)^{<}, \sigma_{p}^{<}$(small green). 
- A real singlet scalar boson $S$ should be introduced as a messenger between the hidden sector and the SM sector, if the hidden sector has fermions and gauge bosons only. Therefore there are two physical Higgs-like scalar bosons.

- There is a destructive interference in the contributions from two scalar bosons in direct detection cross section, which can not be seen in the effective lagrangian approach based on Eq. (2.4).

- Higgs can decay into a pair of CDM, if kinematically allowed, which is begun to constrained by the LHC data.

- Production cross section for Higgs boson is smaller than the SM Higgs boson because of the mixing with composite scalars from the hidden sector.

- Depending on the parameters, only one or none of the two Higgslike scalar boson(s) could be found at the LHC.

- Recent results on the Higgs-like new boson with mass around with $125 \mathrm{GeV}$ from the LHC will constrain this class of models. In particular there is a universal reduction of the signal strength in all the channels. If the future data do not respect this universal suppression, our model would be excluded, independent of discovery of the second Higgs boson.

\section{Acknowledgements}

The author is grateful to Seungwon Baek, Taeil Hur, Dong Won Jung, Jae Yong Lee and WanIl Park and Eibun Senaha for enjoyable collaborations on the subjects reported in this talk and other related issues.

\section{References}

[1] S. Baek, P. Ko and W. -I. Park, JHEP 1202, 047 (2012) [arXiv:1112.1847 [hep-ph]].

[2] S. Baek, P. Ko, W. I. Park and E. Senaha, JHEP 1211, 116 (2012) [arXiv:1209.1685 [hep-ph]].

[3] T. Hur, D. -W. Jung, P. Ko and J. Y. Lee, Phys. Lett. B 696, 262 (2011) [arXiv:0709.1218 [hep-ph]].

[4] P. Ko, Invited talk at the 4th International Conference on Flavor Physics, KITPC, Sep. 24-28, 2007, Beijing. Int. J. Mod. Phys. A 23, 3348 (2008) [arXiv:0801.4284 [hep-ph]]; P. Ko, Invited talk at the Dark Side of the Universe, Melbourne. AIP Conf. Proc. 1178, 37 (2009); Talk at ICHEP 2010, Paris. PoS ICHEP 2010, 436 (2010) [arXiv:1012.0103 [hep-ph]].

[5] T. Hur and P. Ko, Phys. Rev. Lett. 106, 141802 (2011) [arXiv:1103.2571 [hep-ph]].

[6] S. Baek, P. Ko, W. I. Park and E. Senaha, "Higgs portal vector dark matter : revisted," in preparation. 